HEINZ LUDWIG ARNOLD

\title{
Schwierige Grenzübergänge
}

Autor artykułu omawia, na podstawie długoletnich osobistych doświadczeń, trudności związane z niemiecko-niemieckimi spotkaniami literackimi, które cechowała zarówno wola dialogu, jak i próby jego udaremnienia.

Der Beitrag berichtet, auf der Grundlage der langjährigen persönlichen Erfahrungen des Autors, von den Mühen deutsch-deutscher literarischer Begegnungen, die vom guten Willen zu einem Dialog ebenso geprägt waren wie von den Versuchen, diesen zu vereiteln.

The author of the article discusses, on the basis of his many years of personal experience, the difficulties connected with the German-German literary meetings, which were marked by the will of dialogue as well as the attempts to prevent it.

Mein erster Kontakt in die DDR entstand im Sommer/Herbst 1964. Wir hatten das erste Heft TEXT + KRITIK im Februar 1963 herausgebracht, und auf dem Tableau der geplanten Hefte stand auch eines über Anna Seghers. Ihr schrieb ich am 19.6.1964 und bat um einen möglichst unveröffentlichten Text und um die Anschriften von Erwin Strittmatter und Christa Wolf, die ich bitten wollte, über Anna Seghers für TEXT + KRITIK zu schreiben. Da wir damals im Zusammenhang mit der Zeitschrift Lesungen und Vorträge organisierten, lud ich auch Anna Seghers zu einer Lesung oder einem Vortrag in Göttingen ein. Sie antwortete positiv am 18.9.64 und schickte ein signiertes Exemplar ihres Romans Das siebte Kreuz. Doch weder wurde damals etwas aus Lesung und Vortrag noch aus dem Heft. Das erschien erst 1973.

Bis dahin zog sich ein kleiner Wechsel von 9 Briefen über einen neuen Text von ihr und später über ein Gespräch, das ich mit ihr für den NDR führen wollte (seit 1971 machte ich für den Sender regelmäßig große Porträtgespräche mit Schriftstellern). Dem Gespräch stimmte sie anfangs zu, doch mit der 
Zeit wich sie immer mehr aus, zuerst, weil sie sich ,nicht wohl genug“ fühle „und außerdem schlecht über [ihre] Zeit verfügen“ könne, schließlich wollte sie im Januar 1973 nur ,ein einfaches Gespräch [...], aber ohne Stenogramm, Tonband oder dergleichen“. Aber auch dieses Treffen kam nicht zustande.

Damals, 1964, entstand auch die Verbindung zu Christa Wolf, die ich ja um Mitarbeit am Seghers-Heft gebeten hatte. Sie wollte dafür einen in der DDR erschienenen Text zum Siebten Kreuz schicken und bot an, andere Mitarbeiter zu fragen, so zum Beispiel Inge Diersen. Doch, wie gesagt, das Heft über Seghers erschien erst später. Seither aber entwickelte sich eine Korrespondenz mit Christa und ab Ende 1964 auch mit Gerhard Wolf, die erst in den 1990er Jahren einschlief. Ein Plan von mir, der sich dann aber auch zerschlug, war eine Anthologie mit Prosa von DDR-Autoren in der BRD. Am 17.11.1964 schrieb Christa Wolf, sie selbst habe keine kurzen Prosastücke, und die nächste Sache, an der sie saß - offensichtlich Nachdenken über Christa $T$. -, werde wieder 250 Seiten lang. Aber sie empfahl mir als unbedingt wichtige Prosa-Autor(inn)en für diese Anthologie Anna Seghers, Stefan Hermlin, Erwin Strittmatter, Franz Fühmann, Johannes Bobrowski, Eduard Claudius, Arnold Zweig. Und von den Jüngeren Dieter Noll, Bernhard Seeger, Erik Neutsch, Hermann Kant, Max Walter Schulz u.a.

Schon 1967 schlug Gerhard Wolf ein TEXT + KRTIK-Heft über Bobrowski vor, das dann aber trotz einiger Vorarbeiten nicht zustande kam (es kam nach einigen vergeblichen Anläufen erst 2005 heraus). Konkret wurde dann das Heft über Christa Wolf selbst, das 1975 erschien. Dazu hatte ich auch den Lektor des Hinstorff-Verlags, Kurt Batt, eingeladen, der aber abschrieb mit der Begründung: ,[...] es ist zudem nicht so einfach, irgendwelche Expektorationen in der BRD erscheinen zu lassen, wenn sie nicht schon in der DDR erschienen sind“. Bis zu seinem plötzlichen Tod am 20. Februar 1975 korrespondierten wir und tauschten regelmäßig Bücher - etwas länger nur als ein Jahr.

Aus Anlass des geplanten Heftes über Christa Wolf besuchten meine Frau und ich die Wolfs in Ost-Berlin. Und als 1976 Kindheitsmuster erschien, erwiderten die beiden Wolfs den Besuch und blieben ein paar Tage bei uns in Göttingen. Daraus entstand eine freundschaftliche Beziehung, die sich fortsetzte unter anderem auch bei Besuchen anlässlich von zweitägigen Lesungen, zu denen ich, im Vorlauf zur Feier, bei der ich den Weinpreis für Literatur vergab, seit 1981 Autoren aus der DDR und aus der BRD zu uns nach Hause in Göttingen einlud: die beiden Wolfs (Christa bekam den Weinpreis 1986), Günter de Bruyn, Rosemarie Zeplin, Christoph Hein, Helga 
Schubert, Sarah Kirsch, die damals aber schon im Westen lebte, Lothar Baier, Hugo Dittberner, Guntram Vesper, Oskar Pastior, Klaus Modick, Joseph Zoderer u. a. m. Ich erinnere noch sehr lebhaft, wie Christa Wolf bei einer dieser letzten Lesungen aus ihrem Manuskript Sommerstück las, das wir alle mochten, von dem sie in ihrer liebenswerten Bescheidenheit aber meinte, es sei vielleicht doch zu privat; vielleicht hat unser aller Zureden dann aber ein wenig dazu beigetragen, dass sie schließlich dieses Buch doch herausgab.

Es gab mit Gerhard Wolf immer wieder Überlegungen, ja Pläne zu gemeinsamen Herausgaben - was nicht immer leicht zu realisieren war. Ein Buch aber entstand, das nach wie vor ein interessantes Bild vermittelt vom Zustand der DDR kurz vor dem Mauerfall. Ab 1987 bereitete Gerhard Wolf einen umfangreichen Sonderband für TEXT + KRITIK vor über die neuesten literarischen Entwicklungen in der DDR, vornehmlich die Prenzlauer-BergConnection; der Band ist aber erst nach dem Mauerfall 1990 erschienen: Die andere Sprache. Neue DDR-Literatur der 80er Jahre. Darin publizierten wir Texte nahezu aller Autoren dieser besonderen Literaturszene, auch jene, von denen sich später herausstellte, dass sie der Stasi zugearbeitet hatten.

Unmittelbare Kontakte mit DDR-Autoren und Germanisten hatten sich für mich schon im September 1964 auf der Frankfurter Buchmesse bei einem Empfang der DDR im, glaube ich, Hessischen Hof ergeben. Damals war Klaus Köpcke Feuilletonchef oder stellvertretender Feuilletonchef des Neuen Deutschland. Und damals war noch der alte Kämpfer und Romancier Willi Bredel dabei, der einen Monat später starb. Dort lernte ich auch Kurt Böttcher kennen, der in der DDR so etwas wie ein Großherausgeber und Vermittler in Sachen Gegenwartsliteratur wurde. Er verantwortete die Edition monographischer Schriften zu - meist - Schriftstellerinnen und Schriftstellern der DDR und des Exils. Ihn interessierte die - ebenfalls monographische - Zeitschrift TEXT + KRITIK, und er regte einen Austausch unserer Hefte an. Über Böttcher entstanden gute Kontakte zu einigen DDR-Verlagen, die die $T E X T+K R I T I K$-Redaktion fortan regelmäßig mit den Neuerscheinungen der DDR-Literatur versorgten.

Diese TEXT + KRITIK-Redaktion bestand damals im Grunde nur aus Lothar Baier, Wolf Wondratschek und mir. Damals kamen die Hefte noch im Georgi Verlag in Aachen heraus, dessen Verleger ebenfalls vom monographischen Prinzip unserer Zeitschrift überzeugt war (er hatte in seinem Programm, neben Zeitschriften wie Die deutsche Friedhofskultur, die ebenfalls monographische Reihe Die Gartenschönheit). 
Unser Dreiergespann wurde im Frühjahr 1965 von Walter Höllerer zu einem einwöchigen Seminar über Helmut Heißenbüttel ins Berliner Literarische Colloquium, damals noch in der Carmerstraße am Savignyplatz, eingeladen. Wir nutzten den Aufenthalt zu einem (meinem ersten) Besuch in der DDR. Lothar Baier kannte durch Korrespondenz den Lyriker Heinz Kahlau, und ihn besuchten wir - Wondratschek hatte das gerade erschienene TEXT + KRI$T I K$-Heft über Andreas Gryphius eingesteckt, das wir Kahlau mitbringen wollten. Das war ein Fehler - jedenfalls sahen das die Grenzer so. Wondratschek wurde am Übergang Friedrichstraße zu einem speziellen Gespräch mit den DDR-Grenzern eingeladen und musste ihnen drei Stunden Rede und Antwort stehen über diese literarische Konterbande, die solch ein Heft über einen Barockautor wohl barg. Das Heft, in dem drei bis dato ungedruckte lateinische Gedichte von Gryphius standen, die uns der polnische Germanist Marian Szyrocki aus Breslauer Archiven besorgt hatte, wurde argwöhnisch betrachtet: das seien schließlich fremdsprachige Texte, die man erst übersetzen müsse - ob da nicht vielleicht ...

Es blieb ein eindrückliches Erlebnis über die kulturelle Interessiertheit der DDR-Grenzer - an den Besuch bei Kahlau selbst habe ich keine Erinnerung mehr.

Ähnliches erlebte ich elf Jahre später, als ich im April 1976 in Berlin an einem Fernsehgespräch mit Hans Werner Richter und Hans Mayer über Dürrenmatts Israelessay Zusammenhänge teilnahm. Ich schlug Dürrenmatt vor, gemeinsam nach Ostberlin zu gehen - Dürrenmatt war vorher noch nie in der DDR gewesen (und danach auch nicht) -, um dort Christa und Gerhard Wolf zu besuchen. Wieder Übergang Friedrichstraße - ich bekam meine Durchgangserlaubnis nach etwa zehn Minuten; aber Dürrenmatt musste, ohne dass irgendein Grund genannt wurde (freilich bemerkte man eine gewisse Nervosität bei den Grenzern), zwei Stunden lang warten, bis man ihm seinen Pass zurückgab und ihn einreisen ließ. Als wir ein paar Tage später mit meinem Wagen durch die DDR zurück nach Göttingen fuhren (ich hatte FD dorthin zu einer Lesung im Deutschen Theater eingeladen), kurbelte, als wir bei Helmstedt zwar die erste, aber noch nicht die letzte DDR-Hürde hinter uns hatten, Dürri die Scheibe runter, reckte die Faust aus dem Fenster und schrie ein paar Mal: „Scheiß-DDR, Scheiß-DDR“ - bis ich ihm etwas ängstlich sagte, es wäre besser, er warte mit dem Schimpfen noch etwas, denn wir befänden uns noch mitten auf DDR-Hoheitsgebiet. Ihn hatte der Besuch in Ost-Berlin zutiefst verstört. 
Von gravierenderen Behinderungen berichtete mir ein Mitarbeiter des Kritischen Lexikons zur deutschsprachigen Gegenwartsliteratur (KLG), Dr. Hans Wolfschütz. Er war, zusammen mit einem Kollegen, Frank Tichy, im Juni 1981 nach Ost-Berlin gereist, um in dessen Grünauer Haus mit Stefan Heym zu sprechen, über den er einen Essay im $K L G$ schreiben sollte. Beim Grenzübergang Friedrichstraße nahm man Wolfschütz zunächst seinen österreichischen Reisepass ab. Nach anderthalbstündiger Wartezeit wurden beide getrennt verhört. Wolfschütz schrieb dann auf: „Dabei wurde ich unter anderem nach Waffen durchsucht und längere Zeit in einem kleinen Zimmer eingeschlossen, wobei man mir selbst den Zutritt zu einer Toilette verweigerte. Die ganze Prozedur dauerte ca. dreieinhalb Stunden und endete schließlich mit dem Hinweis, man werde uns diesmal die Einreise noch einmal gestatten. Als Grund für diese Behandlung erklärte man uns, dass ,der Tiefdruckstempel in meinem Reisepaß nicht in Ordnung sei“, offensichtlich ein bloßer Vorwand.

Bei ihrer weiteren Reise wurden Tichy und Wolfschütz in der S-Bahn nach Grünau ständig von einem uniformierten Volkspolizisten ,begleitet'. $\mathrm{Zu}$ einem eindeutig inszenierten Zwischenfall kam es dann in der S-Bahn-Station Grünau - Wolfschütz weiter in seinem Bericht, den er mir damals schickte: „Ein Mann in Zivilkleidung, von dem ich vorher genau beobachten konnte, dass er sich mit dem uns ,begleitenden " Volkspolizisten verständigt hatte, lief plötzlich auf uns zu, versetzte Herrn Tichy aus vollem Lauf einen gezielten Tritt gegen den Knöchel des linken Fußes und beschimpfte ihn mit den Worten ,Du blödes Schwein“. Daraufhin verließ er schnell den in der Zwischenzeit zum Stillstand gekommenen Zug und verschwand in der Menge. Der Volkspolizist, der die Szene sehr wohl beobachtet hatte, verhielt sich indifferent, behielt uns aber deutlich im Auge, während wir die Station verließen. Auf dem ca. fünfzehnminütigen Weg zu Herrn Heyms Haus wurden wir schließlich von einer Dame in Zivil beschattet, die sich auch keinerlei Mühe gab, ihre Aufgabe zu verschleiern.“

Als ich 1974 Wolf Biermann besucht hatte, um mit ihm über ein 1975 in der edition text + kritik erschienenes Biermann-Buch zu sprechen, musste ich diesen Besuch auch büßen: auf dem Rückweg zerlegten die DDR-Grenzer meinen neuen K 70 in möglichst viele Einzelteile, und ich hatte, da ich den Wagen noch nicht so gut kannte, dann recht lange damit zu tun, das gute Stück wieder zusammenzubauen.

Anfang der 1970er Jahre bereitete ich für eine von mir herausgegebene, auf 20 Bände angelegte Geschichte der deutschen Literatur aus Methoden für 
den Fischer-Athenäum Taschenbuch Verlag (der dann aber um 1975 seine Arbeit einstellte, von den 20 Bänden erschienen nur fünf) zwei Bände zur Exilliteratur vor. In diesen Bänden sollten auch Texte von Heinrich Mann abgedruckt werden. Die Rechte dafür lagen in der DDR. Ich schrieb deshalb einen Brief an den Aufbau Verlag und bat um die Abdruckrechte. Man antwortete recht schnell und bat darum, eine Übersicht über die in den Bänden erscheinenden Texte einsehen zu dürfen. Ich schickte die Liste, auf der natürlich auch Texte von Alfred Kantorowicz vermerkt waren. Postwendend kam aus Ostberlin die Antwort: Wenn Texte von Kantorowicz in dem Projekt erscheinen würden, werde es keine Erlaubnis zum Abdruck von Texten Heinrich Manns geben. Wütend schrieb ich einen, wie man wohl meinen konnte: naiven Brief (den ich in Kopie an Kurt Böttcher schickte) - Tenor des Briefs: Es sei doch wohl nicht zu glauben, dass ausgerechnet die sozialistische DDR die Politik des nationalsozialistischen Deutschland verlängere, indem sie einen tapferen Mann wie Alfred Kantorowicz, den die Nazis bereits 1933 ausgebürgert hatten, neuerlich aus einem Zusammenhang vertreiben wollte, in den er - gerade mit Heinrich Mann, den er ja ediert hatte - doch ganz genuin gehöre. Das sei ein Skandal, den ich, würde der Aufbau Verlag bei seiner Meinung bleiben, nicht verhindern würde. Ich ahnte, dass ich damit vermutlich die letzte Chance, die Abdruckrechte zu bekommen, verspielt hatte - aber es kam für mich natürlich nicht in Frage, mich dem Diktat der DDR zu beugen und sie mein Programm der Exilbände bestimmen zu lassen. Ich hätte dann die Heinrich-Mann-Texte ohne Genehmigung gedruckt (so mein Verlag mitgespielt hätte) und dazu den Forderungsbrief aus der DDR gestellt.

Es dauerte nicht lange, bis die Antwort auf meinen Brief kam - zwar ging man mit keinem Wort auf meine Argumente in meinen Brief ein, aber man gab mir, als sei nichts geschehen, die Rechte zum Abdruck der Texte Heinrich Manns, ohne jede Einschränkung.

Ich glaube, dieser Erfahrung eingedenk, dass sich manche literarische Beziehung zur DDR offener, klarer und deshalb produktiver hätte gestalten lassen, wenn man in der BRD verständnisvoller und zugleich weniger hasenfüßig, also standfester und entschiedener in den eigenen Plänen mit den Gegenübern in der DDR sich verhalten hätte. 